\title{
"What does it mean?": Uncertainties in understanding results of chromosomal microarray testing
}

\author{
Marian Reiff, PhD, MSc'1, Barbara A. Bernhardt, MS, CGC', Surabhi Mulchandani, MS, CGC2, \\ Danielle Soucier, MBE${ }^{1}$, Diana Cornell, BA ${ }^{1}$, Reed E. Pyeritz, MD, PhD' ${ }^{1}$ and \\ Nancy B. Spinner, PhD 2,3
}

\begin{abstract}
Purpose: The increased sensitivity of chromosomal microarray (CMA) technology as compared with traditional cytogenetic analysis allows for improved detection of genomic alterations. However, there is potential for uncertainty in the interpretation of test results in some cases. This paper explores how families understand and make meaning of CMA test results, and identifies the needs of families undergoing CMA testing.
\end{abstract}

Methods: We conducted semistructured interviews with parents of 25 pediatric outpatients with CMA test results indicating either a pathogenic alteration or a variant of unknown significance (VUS). Interviews were analyzed qualitatively.

Results: Three domains of understanding were identified: comprehension of results, interpretations of scientific uncertainty, and personal meaning for the child and family. Incomplete comprehension

\section{INTRODUCTION}

The increased sensitivity of chromosomal microarray (CMA) technology as compared with traditional cytogenetic analysis allows for improved detection of genomic alterations. Consequently, CMA testing for copy number variation is now recommended as a first-line test for evaluating individuals with a variety of indications, including multiple anomalies not specific to a well-delineated genetic syndrome, apparently nonsyndromic developmental delay/intellectual disability, and autism spectrum disorders. ${ }^{1,2}$ Identification of underlying etiology is important for counseling about prognosis and recurrence risk in the family as well as ending the parental diagnostic odyssey. However, this type of testing also detects many genomic variations of unknown significance, leading to uncertainty in the interpretation of test results. ${ }^{3,4}$ Understanding of genetic test results tends to be poor in the general public ${ }^{5}$ and in individuals undergoing genetic testing to assess disease risk. ${ }^{6} \mathrm{CMA}$ results that involve genomic variations with uncertain clinical courses are especially challenging to understand. ${ }^{78}$ It is therefore important to document how families comprehend and make meaning of the genetic information they receive, and explore ways to improve their understanding.

Microarray testing results fall into three main categories: (i) negative (no clinically significant alteration), (ii) pathogenic of test results and scientific uncertainty were prominent themes for families receiving results in both the VUS and pathogenic categories. Receiving results from non-geneticists and by telephone, long waits to see a geneticist, and misleading Internet searches all contributed to misunderstandings.

Conclusion: Differentiating domains of understanding allows for the identification of uncertainties that can be reduced or managed in order to improve understanding of CMA results. Using this framework, we suggest interventions to promote clarity and address the informational needs of families undergoing CMA testing.

Genet Med 2012:14(2):250-258

Key Words: comprehension; genetic testing; health communication; qualitative; uncertainty

(a genomic alteration known to result in a genetic condition), and (iii) "variant of unknown significance" (VUS) (a deletion/ duplication that has not previously been described, has not been seen in controls so far studied (e.g., the Database of Genomic Variants, http://projects.tcag.ca/variation), and for which there is incomplete data on the genes in the region). Parental testing is recommended for VUS results to determine whether the variant is inherited or de novo. When the parents are asymptomatic (as in most cases), a de novo variant is likely to be pathogenic, and an inherited alteration is likely to be benign. However, parental testing may not completely clarify the clinical significance of the alteration. When a novel de novo change is identified, it is sometimes difficult to predict its contribution to the child's phenotype, and prognosis remains uncertain. In some of the inherited alterations, variable expressivity and incomplete penetrance can increase the uncertainty regarding the clinical significance for the patient and the recurrence risk of the phenotype. It is important to note that some VUS results, such as the 1q21.1 deletion, have been reported in the literature to be associated with highly variable phenotypic features and may or may not provide an etiological explanation for the clinical features prompting the CMA study. ${ }^{9,10}$ Given the background of uncertainty surrounding the medical conditions of children having CMA testing, it

${ }^{1}$ Center for the Integration of Genetic Healthcare Technologies, Division of Translational Medicine and Human Genetics, Perelman School of Medicine, University of Pennsylvania, Philadelphia, Pennsylvania, USA; ${ }^{2}$ Cytogenomics Laboratory, Department of Pathology and Laboratory Medicine, The Children's Hospital of Philadelphia, Philadelphia, Pennsylvania, USA; ${ }^{3}$ Department of Pathology and Laboratory Medicine, Perelman School of Medicine, University of Pennsylvania, Philadelphia, Pennsylvania, USA. Correspondence: Marian Reiff (Marian.Reiff@uphs.upenn.edu) 
is important to understand how families respond to the uncertainties arising from the test results. Previous studies investigating parental uncertainty have focused on families either having or lacking a causal genetic diagnosis for a child's condition., ${ }^{8,11,12}$ Other studies have investigated the effects of uncertainties about prognostic information, illness, cognitive limitations, and life span among families with a child having a specific genetic diagnosis. ${ }^{13-15}$ Although the impact of uncertain genetic test results has been studied in newborn screening ${ }^{16,17}$ and cancer genetics, ${ }^{18,19}$ little research has been conducted on the impact of uncertain CMA test results in the context of pediatric diagnostic testing. Our approach is informed by a conceptual framework proposed by Han et al..$^{20}$ in which understanding of test results can be described and classified in terms of fundamental sources and types of uncertainty. In this article, we describe families' processes of understanding pathogenic or VUS microarray test results. We explore how families understand and make meaning of the results; identify sources of uncertainty that can impede understanding; and elucidate strategies for dealing with uncertainty, promoting clarity, and addressing the informational needs of families undergoing CMA testing.

\section{MATERIALS AND METHODS}

The data described in this article represent one component of an exploratory study of the impact of CMA testing on families and health providers. The study included families and health providers of pediatric outpatients who had CMA testing at the Children's Hospital of Philadelphia. The overall study included clinical observations and pre- and post-test interviews with family members and health providers of children tested, and extended case studies involving multiple interviews with selected families. This paper includes data from a single post-test interview (the first conducted, for those interviewed multiple times) with one or both parents of children with either VUS or pathogenic results. Forthcoming papers will address provider perspectives and psychosocial/ethical issues involved in CMA testing.

\section{Participants}

Participants were interviewed either in person or by telephone using two recruitment strategies.

In-person interviews. We used a convenience sample of families attending outpatient visits. Genetic counselorsidentified families undergoing CMA testing and the researcher (M.R., who was not part of the clinical team) was introduced by the physician. Potential participants had an opportunity to ask questions about the study before providing consent to participate. Parents of eight children were invited, and all agreed to participate in the study. Interviews were conducted at the outpatient clinic, immediately following the medical consultation. The families had already been informed of the CMA results by phone and had discussed results at the consultation.

Telephone interviews. Letters explaining the study and inviting participation were mailed to parents of children who had undergone CMA testing at the Children's Hospital of Philadelphia Cytogenomics Laboratory. In this way, it was possible to recruit families who were referred for CMA testing by different health providers, including non-geneticists. Potential participants were asked to respond if they were interested in participating in a telephone interview and were offered a $\$ 10$ incentive. A follow-up call was made to those who did not respond. Of 44 letters of invitation, 19 parents indicated their willingness to respond, of whom 15 participated in a telephone interview, one was interviewed following an outpatient appointment (counted with the in-person interviews, above), and three were unavailable and did not respond when we attempted to recontact them by telephone. In addition, two parents (one recruited at an outpatient visit and one introduced to the researcher by a genetic counselor) were interviewed by phone, making a total of 17 telephone interviews. An informed consent script was read to potential participants over the phone and oral consent was obtained before the start of each interview.

The study was approved by the Children's Hospital of Philadelphia Institutional Review Board.

\section{Data collection and analysis}

Interviews typically lasted 40-60 min and were conducted by two of the authors (M.R. and D.S.). Using semistructured interview guides, participants were asked a set of open- and closedended questions, with probes to elicit detailed descriptions of their experiences regarding CMA testing. This paper primarily addresses qualitative responses to questions concerning the process of understanding test results, and the meaning of the results, for example, "What is your understanding of what the test results mean in regard to your child's chromosomes?" "How well do you feel like you understand the results?" "What was useful in helping you to understand the results?" and "In general, was there anything you found helpful (or stressful) about having the array testing and getting the results? Probes included "What did you find helpful (or stressful)?" and "Can you explain more about that?" Questions about receiving test results included "Who told you the results?" and "Were you told in person or by phone?" Demographic questions were asked at the end of the interview.

Interviews were audiotaped, transcribed, and entered into NVivo for coding and analysis. ${ }^{21}$ Interviews with both parents of the same child were not considered independent and were analyzed as one family interview. We used a content analysis approach to identify themes and develop a coding scheme. ${ }^{22}$ Codes were generated by the author (M.R.) and five transcripts were independently coded by two additional coders (D.S. and D.C.). Through an iterative process, codes were refined and inconsistencies were resolved by discussion. Ten transcripts were then independently coded by two coders (M.R. and D.S.). The average intercoder agreement was $96.6 \%$. The remaining transcripts were coded by M.R. A matrix was created based on a dichotomous count of codes occurring during each interview (i.e., if a theme occurred in an interview, it was counted once 
regardless of how often that theme occurred). This allowed us to identify occurrences of themes across and within each result category (VUS and pathogenic). ${ }^{22-24}$

\section{Sample characteristics}

This article reports data from interviews conducted with parents of 25 patients who received either pathogenic $(n=11)$ or VUS $(n=14)$ results (Table 1$)$. This sample is not representative of the proportions of pathogenic and VUS results reported by the testing laboratory, which are each $\sim 10 \%$ of tested cases. A total of 25 interviews were conducted with 31 parents (23 mothers and 8 fathers). Of eight in-person interviews, both parents were present at six interviews, only

Table 1 Characteristics of study sample $(n=25)$

\begin{tabular}{|c|c|c|}
\hline Demographic and medical information & $\begin{array}{c}\text { Total } \\
\text { number }\end{array}$ & Percentage \\
\hline \multicolumn{3}{|l|}{ Age of patient (range $=6$ months to 20 years) } \\
\hline$<4$ years & 16 & 64 \\
\hline $5-10$ & 4 & 16 \\
\hline $11-20$ & 5 & 20 \\
\hline \multicolumn{3}{|l|}{ Gender of patient } \\
\hline Male & 13 & 52 \\
\hline Female & 12 & 48 \\
\hline \multicolumn{3}{|l|}{ Ethnicity of patient } \\
\hline White & 23 & 92 \\
\hline African American & 2 & 8 \\
\hline \multicolumn{3}{|l|}{ Ordering physician } \\
\hline Geneticist & 17 & 68 \\
\hline Nongeneticist & 8 & 32 \\
\hline \multicolumn{3}{|l|}{ Test result category } \\
\hline Variant of unknown significance & 14 & 56 \\
\hline Pathogenic & 11 & 44 \\
\hline \multicolumn{3}{|c|}{$\begin{array}{l}\text { Time elapsed between test and interview (months) } \\
\text { (range }=1-24 \text { months) }\end{array}$} \\
\hline$<6$ months & 13 & 52 \\
\hline $7-12$ months & 7 & 28 \\
\hline 13-18 months & 4 & 16 \\
\hline 19-24 months & 2 & 8 \\
\hline \multicolumn{3}{|l|}{ Parent interviewed } \\
\hline Mother & 17 & 68 \\
\hline Father & 2 & 8 \\
\hline Both mother and father & 6 & 24 \\
\hline \multicolumn{3}{|l|}{ Interview method } \\
\hline In person & 8 & 32 \\
\hline Telephone & 17 & 68 \\
\hline \multicolumn{3}{|l|}{ Delivery modality of result } \\
\hline Phone only & 10 & 40 \\
\hline Phone with in-person follow-up & 8 & 32 \\
\hline In person & 7 & 28 \\
\hline
\end{tabular}

the mother at one, and only the father at another. Telephone interviews were conducted with one parent (16 mothers and 1 father) of each of 17 patients. One interview (counted as an in-person interview) was started at the clinic and completed by phone the following day to accommodate the parents time constraints. The sample included patients with a wide range of phenotypes including intellectual disability, multiple congenital anomalies, developmental delay, autism, hypotonia, short stature, seizures, and hearing loss. As many of the genetic variations are extremely rare, specific CMA results are not reported here to prevent disclosing potentially identifying medical or genetic information.

\section{RESULTS}

Incomplete comprehension of test results and a need for more information to improve understanding were prominent issues for parents receiving either a VUS or pathogenic result. The broad theme of understanding was differentiated into three domains: (i) comprehension of results, (ii) interpretations of scientific uncertainty, and (iii) personal meaning for the child and family. Our results are organized according to themes occurring in these domains and statements illustrating themes are presented in Table 2.

\section{Comprehension of results}

We use the term "comprehension" to refer to an individual's self-reported ability to grasp the meaning of the result received. Ten families (five VUS and five pathogenic) reported experiencing difficulty comprehending the result initially. For six of these families (four VUS and two pathogenic), the result was later clarified through additional counseling or information.

Health-care professionals. In our sample, health-care professionals were the primary source of information about the results. The majority of families (15) received results initially from a genetics expert (clinical geneticist or genetic counselor), and all those who were initially informed by non-genetics professionals subsequently had contact with a practitioner with genetics expertise.

Most participants reported that health-care professionals helped them to understand the test results, but several reported receiving inconsistent and/or misleading information. As patients often saw multiple providers, some families were exposed to differing interpretations. Respondents from four families (one VUS and three pathogenic) reported receiving results initially from non-genetics professionals lacking the expertise to provide adequate explanation. This type of misunderstanding was usually resolved eventually by discussion with genetics experts.

Parents did not always rely solely on genetics experts for their most valued information. Several stated that a familiar provider (pediatrician, cardiologist, neurologist, or nurse practitioner) helped most, by explaining the result in the context of the child's medical history and providing support regarding future surveillance. Two families found a team approach (several health professionals meeting with the family in 
Table 2 Themes and illustrative statements pertaining to understanding chromosomal microarray test results Theme Statement

A. Comprehension of results

1. Health-care professionals

- Genetics expertise

- Multiple providers

- Inconsistent information

[We were] just totally devastated by the initial results, ... [the neurologist] told me that I had the same duplication as [my child], and he had never seen that before... But when I went to the genetics department, they told me it's actually very common... So for six months I really didn't have a very good understanding of what was going on ... I didn't think there was any hope. . . . Had I known so many people carry something, I probably would have been a little less stressed. [TMV]

- Team approach

- Familiar health provider

They had everybody. . . . the genetic counselor, my old neurologist, the new neurologist, and the PT. . . So everybody

2. Delivery modality

- Telephone delivery

- Wait for appointment

- Visual materials

- In-person delivery was there together giving us their information on it. [TMP]

3. Internet searches

- Internet-useful

I knew the deletion from when they gave me the results (by phone). . . But I didn't know what they meant. . . I was going crazy trying to figure that out... I wasn't understanding what I was reading [online]. . . I didn't want to wait to get [to the appointment] so I was trying to figure it out on my own. [At the appointment] the charts really helped because I'm more visual. [IMV]

I think what they told us up front was probably the best they could. But I think it was on our part to go and take a look at it and go in more depth. . . I I think we didn't have the full scope of it. [IFV]

- Internet-misleading When I heard it was a deletion on the fourth chromosome I looked it up and it said it can cause heart problems, autism, and [require] special-needs schools. . . I didn't know that [she was missing] only a tiny part. . . it was pretty bad stuff. [IFV]

4. Other informational resources

- Knowledge of genetics (limitations)

I was in tears after looking online for ten minutes and I was convinced he had everything. Sometimes too much information is a bad thing. ... I know my limitations. [IMV]

I didn't go to school for genetics and all that. So I really don't know. And I don't think as a parent I need to know all of that much about it, just what to look forward to, if there are going to be any delays. [TMP]

- Medically informed persons

If I hadn't already sort of known ahead of time what the specific markers they were going to be looking for were, in network and what to request, I think I really would have been confused. But because of the online group that I'm on, I already knew the syndromes that were possible that she could have. [TMV]

B. Interpretations of scientific uncertainty

1. Search for meaning

- Meaning not clear

We had a diagnosis. But then it was just a hundred more questions on top of that. [TMP]

She's missing part of her seventh chromosome. However, they had absolutely no information as to what this meant. [TMP]

There's no concrete information on what the actual duplication means. [TMV]

2. Causal explanation/prognostic information

- Causal explanation It was helpful just to know that that was the reason why. [TMP] It's helpful knowing there's a cause. [IMV]

- Prognostic information It's nice to have the head's up on what could be, or what to look for. [TMP] (helpful)

- Prognostic information (lacking)

We don't know what to expect in the future. [TMP]

- Rarity

It provided answers as to what was causing his delay, but it didn't provide answers as to his future prognosis. Because there are so few other people that have it. So he's a pioneer. [TMP]

- Inconsistent interpretation

The array test gave us the final diagnosis. . . exactly what it was. . . so now we know what to expect and what to plan for, for the future. ... [But] it's such a rare disease. There's not a lot out there. . . so we don't really know what he's going to do in the future. There's a wide spectrum . . . everything from a life expectancy of two to seventy. And I have no idea where he's going to fall in that. [TMP]

3. Scientific knowledge

- Future scientific advancement The only way you're going get more information is if they increase with the technology and more people get it done. [IMV]

IFV (in-person interview, father, VUS); IMV, (in-person interview, mother, VUS); TMP, (telephone interview, mother, pathogenic); TMV, (telephone interview, mother, VUS); VUS, variant of unknown significance.

Table 2 Continued on next page. 
Table 2 Continued.

\begin{tabular}{|c|c|}
\hline $\begin{array}{l}\text { Theme } \\
\text { - Limits of current scientific } \\
\text { knowledge }\end{array}$ & $\begin{array}{l}\text { Statement } \\
\text { There's no concrete information on what the actual duplication means.... [This] genetic testing was really not around } \\
\text { ten years ago. So I guess their limited knowledge is to be expected. [TMV] }\end{array}$ \\
\hline \multicolumn{2}{|l|}{ 1. Relief } \\
\hline $\begin{array}{l}\text { - Relief at finding a causal } \\
\text { explanation }\end{array}$ & $\begin{array}{l}\text { It's [helpful] knowing that there is a cause behind the problems that [my child] is having, so when I'm shuffling him off } \\
\text { people don't think I'm insane for taking him to all the different places because he really does have [a deletion.] [IMV] }\end{array}$ \\
\hline - Alleviation of guilt or blame & $\begin{array}{l}\text { Every single time they [health providers] would dig into my gynecological history. . . . I didn't drink. I didn't smoke. } \\
\text { I didn't do drugs. .. . I tried to do everything right. Having this test was finally the answer that she was wired this } \\
\text { way. . . This really was the hand that God himself dealt us. [TMV] }\end{array}$ \\
\hline \multicolumn{2}{|l|}{ 2. Self-concept } \\
\hline $\begin{array}{l}\text { - Access to services } \\
\text { - Empowerment }\end{array}$ & $\begin{array}{l}\text { It might be a tiny little segment of a chromosome, but it's a big frickin' stick when I walk back in that next IEP } \\
\text { [Individual Educational Plan] meeting ... It has empowered us. [TMV] }\end{array}$ \\
\hline $\begin{array}{l}\text { - Need for connection with } \\
\text { other families }\end{array}$ & $\begin{array}{l}\text { It [would] mean the world that somebody else somewhere out there has seen a [child like mine] ... that we're not } \\
\text { alone. [TMV] } \\
\text { It's really hard when you walk away with no information. If they could just say... "Here's a number of a support } \\
\text { group". . like "Hey, there's another step. It just doesn't end here." [TMV] } \\
\text { They couldn't give me any information on anyone else. I said "please, if you can, you could give them my } \\
\text { information," but it was just a shut door. [TMP] }\end{array}$ \\
\hline
\end{tabular}

IFV (in-person interview, father, VUS); IMV, (in-person interview, mother, VUS); TMP, (telephone interview, mother, pathogenic); TMV, (telephone interview, mother, VUS); VUS, variant of unknown significance.

person) helpful in aiding comprehension of test results and planning medical care.

Delivery modality. The delivery modality (telephone or in person) influenced comprehension of the results for some participants. Three respondents reported that their comprehension was impeded due to receiving results by telephone and was substantially improved by a subsequent in-person consultation. Two respondents who received results only by telephone would have liked more in-depth discussion in person but had difficulty getting to the hospital for a consultation. Another mentioned that the information conveyed in person was more detailed than information conveyed by phone. Visual materials (available only in the in-person consultations) were reported to facilitate comprehension. Several respondents reported that they benefited from the presence of multiple professionals (including geneticists, genetic counselors, and other health professionals) at an in-person consultation. One mother reported that her husband received the result by phone and had difficulty relaying the information to her. In-person consultations provided an opportunity for both parents and/or additional family members to be present to hear the explanations and raise questions about the results. In one interview, the father had a preference for visual and written materials, whereas the mother preferred auditory information, and, as a family, they expressed the need for both types of information.
Long waiting periods between receiving results by phone and in-person consultations can cause misunderstandings to linger and become compounded. For example, a misunderstanding of information conveyed by phone led one mother to think that her daughter would be infertile, and while awaiting an appointment with a clinical geneticist, she found additional misleading information on the Internet about severe medical complications. At the consultation, she learned that her worries were unfounded.

Internet searches. Most respondents supplemented the information they received from their health providers with other resources, primarily the Internet. Respondents' experiences with seeking information on the Internet were mixed. For most, Internet searches did not yield much useful information. A few respondents reported that they did not conduct Internet searches because they were advised by clinicians that no information was available. Some respondents (four) found Internet searches helpful and an equal number (four) reported finding misleading and/ or anxiety-provoking information. Searches were helpful in clarifying medical terms and in seeking information using specific, detailed results. Several respondents reported finding disturbing information on the Internet about extremely severe cases.

A recurring theme in our data was conducting Internet searches based on incomplete information about the result, for example, not including the specific location and size of the deletion/duplication on the chromosome. Information gleaned 
from these searches aroused needless anxiety, until clarified by a genetics expert. A typical example is a couple warned by their physician not to search the Internet. Although the mother heeded this advice, the father conducted a search, finding disturbing information relevant to the deletion of the entire chromosome, rather than the microdeletion present in his child.

Other informational resources. Respondents found that limitations in their own knowledge of genetics impeded their comprehension of test results and limited their ability to gather information from a variety of sources. Some, who recognized their limitations, decided not to search on their own, but to rely solely on health professionals for information and guidance.

Comprehension was aided for some by medically informed relatives or friends. One parent attributed her understanding of the results to being well-informed about microarray testing prior to testing through her connection with an online community based on her child's clinical diagnosis.

\section{Interpretations of scientific uncertainty}

Uncertainty was introduced when it was unclear if the result provided a causal explanation for the patient's clinical features or if prognosis and future medical needs could not be reasonably predicted.

Search for meaning. Many participants, even if they comprehended the result, still struggled to understand the meaning and potential implications of results and expressed a need for more information and explanation. A typical response was "I understood what they told me, but I wish we could have found out more about what it meant ... I wanted to know more." (in-person interview, mother, VUS)

Causal explanation/prognostic information. Respondents from 13 families (8 pathogenic and 5 VUS) reported that the result provided a causal explanation. In some cases, prognostic information was available. Respondents from eight families (four pathogenic and four VUS) articulated that the result might help to anticipate their child's future needs, for example, a need for medical monitoring of heart or kidney functioning. However, in many cases of newly defined pathogenic deletions or duplications, very little information was available about prognosis because of rarity, variable expressivity, and the fact that the disorders are newly identified. Although these parents did experience some closure to the search for answers, many continued to experience considerable uncertainty regarding the future course of their child's health and development. Participants sometimes held inconsistent interpretations, on one hand, that the CMA result helped them to know what to expect, and on the other, acknowledging the lack of prognostic information.

Scientific knowledge. A common perspective was that the benefits of the test would become more apparent in the future, as more people are tested. The participants were generally committed to being part of a continuing search and hopeful that future scientific advancement would provide more answers about their child's medical condition. For many, an important part of the understanding process was to clarify the scientific uncertainty inherent in the result and acknowledge the limits of current scientific knowledge.

\section{Attributions of personal meaning}

In addition to accurately comprehending the result and appreciating the scientific uncertainties involved, parents also work to understand the meaning and implications of the result for their child, themselves, and their family. Personal meanings encompass psychological and emotional processes including relief, changes in self-concept, and coping resources.

Relief. A sense of relief at finding a causal explanation was expressed primarily by parents receiving pathogenic results and also by some with VUS results. On the other hand, some participants expressed relief at not receiving a definitive result, associated with the view that a genetic anomaly is irreparable. Although a negative or inconclusive result does not in fact rule out the possibility of a genetic problem, one participant articulated the belief that it did and was therefore relieved that a definitive pathogenic result was not found.

The understanding that the test result provided a causal explanation allowed some respondents (in both the VUS and pathogenic groups) to alter their previously held notions about possible causes of their child's disorder. For some, this meant the alleviation of the burden of guilt and blame for having contributed to the disorder during the pregnancy, delivery, or infancy of the child.

Self-concept. One parent reported being shocked to discover, following parental testing, that he himself was carrying the same deletion found in his child, which may be associated with a wide range of disorders and is also found in healthy individuals. The finding led him to question his sense of self and raised concerns about the potential for stigma and discrimination at work.

Coping resources. Several respondents reported that they found the results (both VUS and pathogenic) valuable and empowering in terms of accessing resources, especially educational services.

Connection with other families with the same genetic variant was mentioned as a potential resource for emotional support as well as factual information, and several parents felt that their experience could have been improved had they been provided more information about resources for family support. Two participants were actively searching for others with the same CMA result and had started Facebook pages with this goal. They both expressed frustration that the hospital could not facilitate a connection with other parents or make their contact information available to others because of privacy regulations.

\section{DISCUSSION}

We investigated parental understanding of CMA test results for children who were diagnosed with either a pathogenic 
alteration or a VUS. Three domains of understanding were identified: (i) comprehension of results, (ii) interpretations of scientific uncertainty, and (iii) personal meanings for the child and family. Receiving results by telephone, long waits to see a geneticist to discuss results, inconsistent information provided by different professionals, and Internet searches based on inadequate and/or inaccurate information all contributed to difficulties in comprehending results. We suggest that these can be addressed to reduce uncertainty and improve parents' understanding of test results.

Some respondents preferred to receive results in person because a personal encounter allowed more in-depth discussion, the presence of multiple practitioners and family members, and the availability of visual materials. This is consistent with research in other medical genetics settings demonstrating increased satisfaction when patients have an option of receiving results by telephone or in person. ${ }^{25,26}$ Comprehension was also improved by discussing results with health providers (geneticists, genetic counselors, trusted non-genetics professionals, and a multispecialist team) and with medically informed relatives, friends, or support groups. Many of these practical issues are addressed in the Recommendations section below.

Consistent with a study by Makela et al. ${ }^{8}$ on parental responses to CMA testing for intellectual disability, we found that Internet searches had limited value and sometimes increased confusion. Rare CMA results may consist only of a chromosomal address or location of a deletion/duplication, rather than the name of a syndrome. Often, parents were aware only of the number of the chromosome with the deletion/duplication, rather than the exact chromosomal address, breakpoints, and size. When searching the Internet without sufficiently detailed information, there is a tendency to find information about extremely severe conditions (e.g., very large deletions or insertions) that increases confusion and anxiety. Although some parents were warned by providers not to search online, many conducted searches anyway. There is a need for health professionals to explain specific details about the result, warn parents about the likelihood of finding inapplicable information on the Internet, and direct them to appropriate websites. Although many searches using the detailed test results may not be fruitful, this would at least avoid the needless distress that occurs when finding disturbing information that is not relevant. By reducing the negative outcomes associated with Internet searches, the Internet may become a valuable resource for information and/or support, as reported in several other studies. ${ }^{17,27-29}$

Recipients of results in both the VUS and the pathogenic categories expressed similar views regarding understanding the scientific uncertainties pertaining to the test results. This was somewhat surprising in light of research suggesting that parents of undiagnosed children and individuals with inconclusive results, as opposed to those with a definite diagnosis, experience greater stress and additional challenges in understanding results of genetic testing. ${ }^{16,17,19,30-33}$ However, for both categories of CMA results, there are often similar uncertainties regarding prognosis. Many pathogenic findings involve rare and newly identified genomic disorders. ${ }^{34}$ Parents of children with rare disorders are known to have high levels of perceived uncertainty due to limited prognostic information and variability of the clinical phenotypes. ${ }^{8,13}$ The line between some of the VUS results and the novel pathogenic results is not always clear, and some VUS results may, after more scientific investigation, become defined as novel syndromes. This may also explain why some parents in the VUS group reported finding a causal explanation for their child's condition.

Although there are some similarities between the VUS and pathogenic groups in understanding of results, research has found that having an inconclusive, rather than a pathogenic, result can lead to different psychological and emotional responses, such as allowing for hope to be maintained. ${ }^{11-13,15,17}$ It is important for future research to identify aspects of genetic variations that may lead to differing emotional responses. The reshaping of identity in response to genetic information has been discussed in research regarding presymptomatic and diagnostic genetic testing. ${ }^{16,35,36}$ In the context of CMA testing, finding a genetic anomaly in parents may alter their self-concept if they discover that they carry the same copy number variation as other individuals who exhibit pathological features. Although beyond the scope of this article, it is important to consider the occurrence of incidental findings in the patient and other relatives tested. These types of unintended consequences will no doubt become more common as genome-wide testing becomes more widely available. ${ }^{32}$ Future research should address the ethical and psychosocial implications of CMA testing for asymptomatic relatives of patients tested, with respect to risk for disease, reproductive decisions, self-concept, and discrimination.

An important contribution of this study is the approach to clarifying different types of uncertainties and identifying those that can and cannot be reduced. Comprehension of test results can be improved by reducing uncertainties attributed to limitations in providers' and family members' personal knowledge of genetics, and improving the quality of communication about the results. Although the uncertainties stemming from limitations in scientific knowledge can only be resolved by future discoveries, it may be valuable for families to differentiate between different types of scientific uncertainties: for example, some pathogenic results provide a causal explanation but unclear prognosis because of the rarity of the finding; the implications of some VUS results may be unclear now, but future research may shed light on the impact of the copy number variation on the child's health and development. When uncertainties are differentiated, families' specific informational needs can be addressed more effectively. Scientific uncertainties that cannot be reduced at present may be addressed first by acknowledging them clearly and then by keeping abreast of relevant medical advances through appropriate follow-up with health providers and by participation in research studies, when available. 
Increased understanding and knowledge may facilitate coping for parents of a child with a rare genetic disorder, ${ }^{6,29}$ and health providers have an important role in fostering skills that may help parents adapt and cope with the uncertainty and stress of their child's condition. ${ }^{13,37}$ Our findings suggest that health professionals can help families to interpret and find meaning in their results by clarifying uncertainties that can be reduced and providing tailored information to address individual needs. Involving primary-care practitioners can be an effective means of providing care to children with special health-care needs. ${ }^{38}$ This highlights the need for continuing genetic education for primary-care physicians so that they can provide care and guidance for families undergoing CMA testing. ${ }^{39}$

\section{Recommendations}

Based on our findings, we make the following recommendations so as to improve the understanding process for families of children having CMA testing:

- Results should be provided by professionals with appropriate genetics expertise to accurately interpret and explain results, as articulated in recent professional guidelines. ${ }^{40}$

- Pre-test education and counseling is needed, and written materials need to be developed, to prepare families for potential diagnostic and prognostic uncertainties.

- Families should be provided with options for the mode of result delivery, either by telephone or in person.

- Post-test genetic counseling should be available soon after results are delivered, to address the medical and psychosocial implications of CMA results for the child and other family members, and inform families of how to keep abreast of new relevant scientific information.

- Resources need to be developed to enable parents to access reliable information and connect with other families with the same CMA finding.

- Guidance should be provided to families using Internet searches, in order to avoid finding misleading information.

- Genetics training for non-genetics specialists and primary health-care providers can help them to provide appropriate support for families and address the medical and psychosocial implications of test results.

\section{Limitations}

There are several limitations to this study. First, we used a small convenience sample and generalizability is constrained. As our intention was to explore understanding of uncertain and abnormal results in the novel context of CMA testing, we excluded families with negative results. This allowed us to maximize our potential to investigate the impact of the results, but the sample is not representative of all families having CMA testing. Second, the different methods of recruitment yielded different response rates, and more fathers were recruited for in-person interviews than telephone interviews. Third, in this retrospective study, recollections of receiving results may be subject to recall bias. Fourth, the children tested varied in age and had a wide range of medical conditions, both of which are likely to influence understandings of the test results. It is important for future research to investigate understandings of CMA results among families with negative test results, specific phenotypes and genotypes, and different sociodemographic backgrounds.

\section{CONCLUSION}

The findings of this exploratory research provide insights into the process of understanding VUS and pathogenic CMA test results for parents of children tested. Three domains of understanding were identified: comprehension of results, interpretations of scientific uncertainty, and personal meanings of the test results for the child and family. Within each domain, different types of uncertainties can be differentiated. The value of distinguishing different types of uncertainties lies in the ability to identify and address sources of uncertainty that can be reduced. Although scientific uncertainty will not be reduced by currently available information, it is possible to reduce other aspects of uncertainty and thereby improve families' understanding of the results. Providing timely, in-person consultations to discuss results can help to reduce uncertainties and promote clarity. Providers need to be aware of families' subjective understandings with respect to comprehension, scientific uncertainty, and personal meanings associated with CMA results. Our findings demonstrate the applicability of a conceptual model of uncertainty to the context of CMA testing and suggest strategies to address the informational needs of families based on the specific types of uncertainties they experience.

\section{ACKNOWLEDGMENTS}

This study was funded by the National Human Genome Research Institute of the National Institutes of Health (supplement PA-04126 to Penn CIGHT grant P50 HG004487). The authors are grateful to the physicians at the Children's Hospital of Philadelphia for granting access to patients and families and to all the individuals (patients, parents, and physicians) who participated in this research.

\section{DISCLOSURE}

The authors declare no conflict of interest.

\section{REFERENCES}

1. Manning M, Hudgins L. Array-based technology and recommendations for utilization in medical genetics practice for detection of chromosomal abnormalities. Gen Med 2010;12:742-745.

2. Miller DT, Adam MP, Aradhya S, et al. Consensus statement: chromosomal microarray is a first-tier clinical diagnostic test for individuals with developmental disabilities or congenital anomalies. Am J Hum Genet 2010;86:749-764.

3. Kohane IS, Masys DR, Altman RB. The incidentalome: a threat to genomic medicine. JAMA 2006;296:212-215.

4. Redon $\mathrm{R}$, Ishikawa $\mathrm{S}$, Fitch $\mathrm{KR}$, et al. Global variation in copy number in the human genome. Nature 2006:444:444-454.

5. Condit CM. Public understandings of genetics and health. Clin Genet 2010;77:1-9.

6. Klitzman RL. Misunderstandings concerning genetics among patients confronting genetic disease. J Genet Couns 2010: 19:430-436. 
7. Darilek S, Ward P, Pursley A, et al. Pre- and postnatal genetic testing by array-comparative genomic hybridization: genetic counseling perspectives. Genet Med 2008;10:13-18.

8. Makela NL, Birch PH, Friedman JM, Marra CA. Parental perceived value of a diagnosis for intellectual disability (ID): a qualitative comparison of families with and without a diagnosis for their child's ID. Am J Med Genet A 2009;149A:2393-2402

9. Mefford HC, Sharp AJ, Baker $C$, et al. Recurrent rearrangements of chromosome 1q21.1 and variable pediatric phenotypes. N Engl J Med 2008;359:1685-1699.

10. Girirajan S, Eichler EE. Phenotypic variability and genetic susceptibility to genomic disorders. Hum Mol Genet 2010;19(R2):R176-R187.

11. Rosenthal ET, Biesecker LG, Biesecker BB. Parental attitudes toward a diagnosis in children with unidentified multiple congenital anomaly syndromes. Am J Med Genet 2001;103:106-114.

12. Lenhard W, Breitenbach E, Ebert $H$, Schindelhauer-Deutscher HJ, Henn W. Psychological benefit of diagnostic certainty for mothers of children with disabilities: lessons from Down syndrome. Am J Med Genet A 2005;133A:170-175.

13. Lipinski SE, Lipinski MJ, Biesecker LG, Biesecker BB. Uncertainty and perceived personal control among parents of children with rare chromosome conditions: the role of genetic counseling. Am J Med Genet C Semin Med Genet 2006;142C:232-240.

14. Van Riper M. Genetic testing and the family. J Midwifery Womens Health 2005:50:227-233.

15. Whitmarsh I, Davis AM, Skinner D, Bailey DB Jr. A place for genetic uncertainty: parents valuing an unknown in the meaning of disease. Soc Sci Med 2007;65:1082-1093.

16. Timmermans $S$, Buchbinder $M$. Patients-in-waiting: living between sickness and health in the genomics era. J Health Soc Behav 2010;51:408-423.

17. Tluczek A, Chevalier McKechnie A, Lynam PA. When the cystic fibrosis label does not fit: a modified uncertainty theory. Qual Health Res 2010;20: 209-223.

18. Baty BJ, Dudley WN, Musters A, Kinney AY. Uncertainty in BRCA1 cancer susceptibility testing. Am J Med Genet C Semin Med Genet 2006;142C: 241-250.

19. Ardern-Jones A, Kenen R, Lynch E, Doherty R, Eeles R. Is no news good news? Inconclusive genetic test results in BRCA1 and BRCA2 from patients and professionals' perspectives. Hered Cancer Clin Pract 2010;8:1.

20. Han PKJ, Klein WMP, Arora NK. Varieties of uncertainty in health care: a conceptual taxonomy. Med Decis Making 2011;31:828-838.

21. Bazeley P. Qualitative Analysis with Nvivo. Sage Publications: Thousand Oaks, CA, 2007.

22. Bernard HR. Qualitative data analysis I: text analysis. In: Research Methods in Anthropology (Qualitative and Quantitative Approaches), 3rd edn. AltaMira Press: Walnut Creek, CA, 2002:440-488.
23. Miles MB, Huberman AM. Qualitative Data Analysis: An Expanded Sourcebook, 2nd edn. Sage: Thousand Oaks, CA, 1994.

24. Morse JM. Evolving trends in qualitative research: advances in mixedmethod design. Qual Health Res 2005;15:583-585.

25. Fanos JH, Gronka S, Wuu J, Stanislaw C, Andersen PM, Benatar M. Impact of presymptomatic genetic testing for familial amyotrophic lateral sclerosis. Genet Med 2011:13:342-348.

26. Baumanis L, Evans JP, Callanan N, Susswein LR. Telephoned BRCA1/2 genetic test results: prevalence, practice, and patient satisfaction. J Genet Couns 2009; 18:447-463.

27. Schaffer R, Kuczynski K, Skinner D. Producing genetic knowledge and citizenship through the Internet: mothers, pediatric genetics, and cybermedicine. Sociol Health IIIn 2008;30:145-159.

28. Skinner D, Schaffer R. Families and genetic diagnoses in the genomic and internet age. Infants Young Child 2006;19:16.

29. Gundersen T. 'One wants to know what a chromosome is': the internet as a coping resource when adjusting to life parenting a child with a rare genetic disorder. Sociol Health Illn 2011;33:81-95.

30. Maheu C, Thorne S. Receiving inconclusive genetic test results: an interpretive description of the BRCA1/2 experience. Res Nurs Health 2008:31:553-562

31. Tabor HK, Cho MK. Ethical implications of array comparative genomic hybridization in complex phenotypes: points to consider in research. Genet Med 2007;9:626-631.

32. Jackson L, Pyeritz RE. Molecular technologies open new clinical genetic vistas. Sci Trans/ Med 2011;3:65ps2.

33. Domchek S, Weber BL. Genetic variants of uncertain significance: flies in the ointment. J Clin Onco/ 2008;26:16-17.

34. Cooper GM, Coe BP, Girirajan S, et al. A copy number variation morbidity map of developmental delay. Nat Genet 2011;43:838-846.

35. Finkler K. Experiencing the New Genetics: Family and Kinship on the Medical Frontier. University of Pennsylvania Press: Philadelphia, PA, 2000.

36. Klitzman R. "Am I my genes?": Questions of identity among individuals confronting genetic disease. Genet Med 2009;11:880-889.

37. Biesecker BB, Erby L. Adaptation to living with a genetic condition or risk: a mini-review. Clin Genet 2008;74:401-407.

38. Rosenthal TC. The medical home: growing evidence to support a new approach to primary care. J Am Board Fam Med 2008;21:427-440.

39. Guttmacher AE, Porteous ME, Mclnerney JD. Educating health-care professionals about genetics and genomics. Nat Rev Genet 2007;8: 151-157.

40. Kearney HM, Thorland EC, Brown KK, Quintero-Rivera F, South ST; Working Group of the American College of Medical Genetics Laboratory Quality Assurance Committee. American College of Medical Genetics standards and guidelines for interpretation and reporting of postnatal constitutional copy number variants. Genet Med 2011;13:680-685 